\title{
A study of small sized quartz tuning fork using finite element analysis
}

\author{
Gwo-Chung Tsai \\ Department of Mechanical and Electro-Mechanical Engineering, National I-Lan University, I-Lan, Taiwan, R.O.C
}

\begin{abstract}
This is a preliminary study to small sized quartz tuning fork operating at $32.768 \mathrm{kHz}$ for several types of supporting structure. Tine length and tine width can be calculated by Euler-Bernoulli equation (Exact Solution), but the base shall be analyzed by FEM (Approximation Solution) due to flexibility of base design. Finite Element Method (FEM) software ANSYS is used to simulate quartz tuning fork to extract electric response from harmonic analysis. Several types of supporting structure are to compare to modified tuning fork with notch by simulation.
\end{abstract}

\section{Introduction}

The design of quartz tuning fork was early treated as Ushape model, and analysis method has been derived from Transfer Matrix Method by J.A. Kusters and...et al. in 70s. The model (Fig. 1) was divided into several segments, and each segment was assumed to operate flexure and length-extensional mode then to extract characteristic frequency of system [1]. The base of quartz tuning fork in the above method didn't include rotary inertia effects. In 90s, Hideaki Itoh and...et al. also took quartz tuning fork to be U-shape model, and the right half section similar to L-shape was composed of three bars then to calculate the characteristic frequency of system by Sezawa's approximation [2]. But in the base, the frequency equation was considered as flexure modes. Based on the assumption of EulerBernoulli equation, the equation should be suitable for long bar not for short bar (Tuning Fork Base). In $80 \mathrm{~s}$, the frequency equation of the flexure modes including rotary inertia effect was introduced by Shih S. Chuang [3], and the frequency equation including rotary inertia effect is suitable for small ratio of tine length to tine width and for higher frequency tuning fork. The quartz tuning fork using overtone flexure modes was developed by Shih S. Chuang.

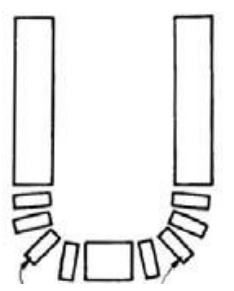

Fig. 1. U-shape tuning fork model [1].

In order to improve the electric characteristic of quartz tuning fork, Yoshiro Tomikawa[4][5] brought up ideas of modified base-width and arm corners cut off (Fig. 2). Those ideas were used in the real products indeed to enhance better electric characteristic, such as a higher Quality factor and lower resistance...etc. In 2003, Fumitaka Kitamura [6] patented a new base shapenotch which is located between tines and mounting area (Fig. 3) in order to get well electric characteristic in miniaturization of quartz tuning fork unit.

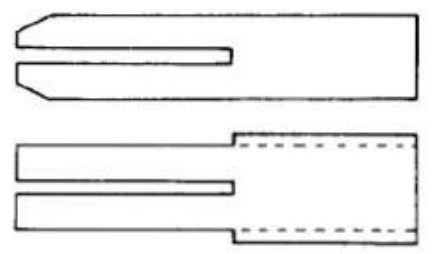

Fig. 2. Modified tuning fork with arm corners cut off and basewidth [5].

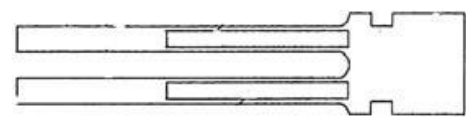

Fig. 3. Modified tuning fork with notch [6].

As known, quartz tuning fork unit has been used in many fields especially in wristwatch for a long time. The dimension of tuning fork unit was not most important issue due to capacious space of wristwatch. However the issue of dimension of tuning fork unit was more important because the growth of mobile phone marketing and the demand of multi-function of consume electronic products was getting strong in the past decade. The quartz tuning fork unit is not only required to meet the tight space but also required to hold the well electric characteristic. Nowadays, the popular packaged dimension of tuning fork unit which used in mobile phone is $4.1 \times 1.5 \mathrm{~mm}^{2}$ and $3.2 \times 1.5 \mathrm{~mm}^{2}$. Recently, the smallest quartz tuning fork unit $2.0 \times 1.2 \mathrm{~mm}^{2}$ has been developed by competitors. Those quartz tuning fork units

\footnotetext{
Corresponding author:gctsai@niu.edu.tw
} 
are much small than before. $\mathrm{mm}^{2}$ has been developed by competitors. Those quartz tuning fork units are much small than before.

For design of small sized quartz tuning fork unit, both tine length and base length shall be decreased to meet tight space. As there is a relationship between length of tine and width of tine governed by EulerBernoulli equation[7] operating at $32.768 \mathrm{kHz}$, the decrease of tine length is limited. So, we are focusing on the analyses of base shape to get proper shape and dimension of base in order to meet requirement of tight space and good electric characteristic. Quartz tuning fork have been widely used in sensor, AFM and gyroscope [8-11].

In this paper, the design of tuning fork is to first extract tine width and tine length from Euler-Bernoulli equation with clamped-free boundary condition, then uses FEM software (ANSYS, Inc.) to simulate quartz tuning fork. The package dimension is $3.2 \times 1.5 \mathrm{~mm}^{2}$ with inner dimension $2.45 \times 0.8 \mathrm{~mm}^{2}$. Several types of supporting structure of quartz tuning fork unit shall be demonstrated to compare to modified tuning fork with notch (Fig.3).

\section{Euler-Bernoulli equations}

The Euler-Bernoulli equation [7] and its frequency equation with clamped-free boundary condition is given by

$$
\begin{gathered}
\frac{\partial^{4} y}{\partial x^{4}}+\frac{1}{a^{2}} \frac{\partial^{2} y}{\partial t^{2}}=0, \text { where }^{2}=\frac{E I}{\rho A} \\
\operatorname{Cos} \beta|\operatorname{Cos} \beta|+1=0
\end{gathered}
$$

$E$ is constant derived from anisotropic elastic constants, and it depends on the cutting angle. $\beta$ is wave number and 1 is beam length. The first root, which means fundamental mode, of (2) is 1.875 . The frequency equation function of tine width and tine length is given by

$$
f=k \frac{w}{l^{2}}
$$

where $\mathrm{f}$ is frequency $(\mathrm{Hz}), \mathrm{k}$ is constant in a specified cutting angle, $w$ is tine width and 1 is tine length.

\section{Improvement by modified base}

As decrease of base length, the electric characteristic of quartz tuning fork is getting poor. It is better that ratio of base length to tine length is larger than $40 \%$ [6]. According to this condition, of which ratio of base length to tine length is equal to $40 \%$, and formula (3) which operates at $32.768 \mathrm{kHz}$, tine width, tine length and base length can be tabularized as Table 1 .
Table 1. Dimensions of specimens.

\begin{tabular}{|c|c|c|}
\hline Tine width & Tine length & Total length \\
\hline 0.05 & 1.165 & 1.63 \\
\hline 0.06 & 1.276 & 1.79 \\
\hline 0.07 & 1.378 & 1.93 \\
\hline 0.08 & 1.473 & 2.06 \\
\hline 0.09 & 1.563 & 2.19 \\
\hline 0.1 & 1.647 & 2.31 \\
\hline 0.11 & 1.728 & 2.42 \\
\hline 0.12 & 1.805 & 2.53 \\
\hline 0.13 & 1.878 & 2.63 \\
\hline 0.14 & 1.949 & 2.73 \\
\hline
\end{tabular}

As ordinary wafer thickness using in quartz tuning fork is $0.1 \mathrm{~mm}$ and tine thickness larger than tine width may cause unwanted vibrating displacement which occurs in not main vibration direction [12], the tine width small than $0.09 \mathrm{~mm}$ to be not discussed this time. The tine width large than $0.12 \mathrm{~mm}$ is also to be not discussed due to total length over inner space. Tine width $0.1 \mathrm{~mm}$ is picked up for case study.

The model of modified tuning fork with base-width (Fig. 3) is to verify that it is better ratio of base length to tine length is larger than $40 \%$ by simulation, the result (Fig. 4) shows the electric characteristic is descent once the ratio is small than $40 \%$.

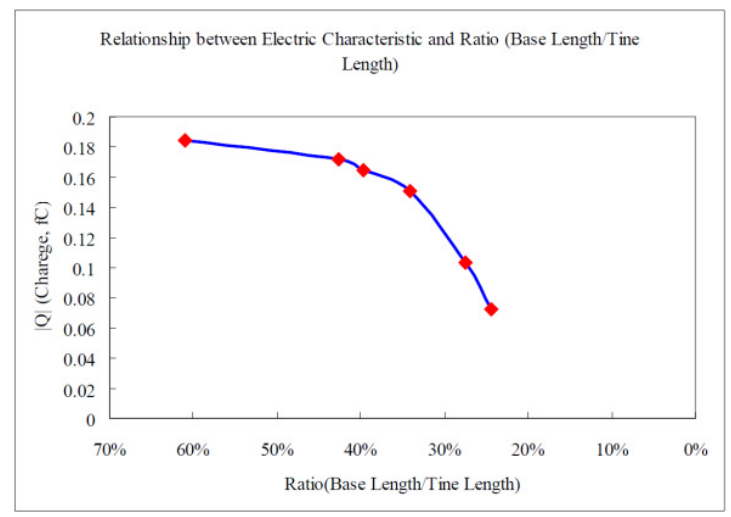

Fig. 4. Relationship between electric characteristic and ratio (Base length/Tine length).

In order to overcome poor electric characteristic due to ratio less than $40 \%$, Fumitaka Kitamura ${ }^{[6]}$ patented a new base shape "notch" which is located between tines and mounting areas. The improvement caused from notch was also verified under ratio (Base length/Tine length) $40 \%$ by simulation as shown in Fig. 5. It is obvious that modified tuning fork with notch has better electric characteristic as vibration energy doesn't leak to mounting areas. 


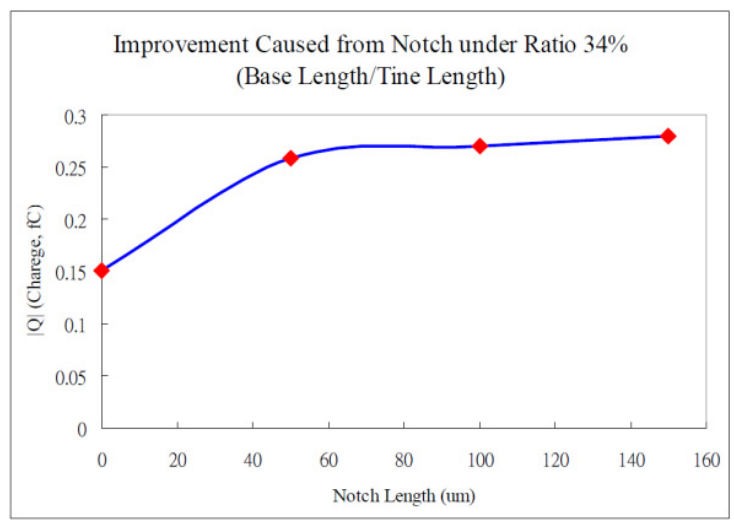

Fig. 5. Improvement resulted from notch under ratio $34 \%$ (Base length/Tine length).

\section{Simulation}

The improvement by modified base comes out and idea. If one can clearly understand the mechanism of supporting structure for vibration energy trapping, small sized quartz tuning fork unit can be realized. Based on the idea [8], several types of supporting structure are built to compare to modified tuning fork with notch. Equations should be centred and should be numbered with the number on the right-hand side.

\subsection{Procedure, conditions and modelling}

Tine length and tine width calculated by Euler-Bernoulli equation at $+2^{\circ} \mathrm{X}$-cut (Operating at $32.768 \mathrm{kHz}$ ) are $1.64 \mathrm{~mm}$ and $0.1 \mathrm{~mm}$ respectively. The thickness of tuning fork model is $0.1 \mathrm{~mm}$. The finite element analysis software ANSYS is used to simulate quartz tuning fork resonator.

\subsubsection{Procedure and conditions}

Modal analysis is first to extract the characteristic frequency then to proceed with harmonic analysis for electric response (Charge per node, $|\mathrm{Q}|, \mathrm{fC}$ ).

a. Average number of element is 4000 .

b. Average number of node is 22400 .

b. Damping ratio is 0.0001 .

c. The comparison is based on electric response of each model calculated by harmonic analysis.

\subsubsection{Model}

The model material is pure quartz crystal without electrode, and voltage as boundary condition applies on specified area on tines. There are five kinds of model regarding various base lengths to be analyzed (Table 2, Fig.6 Fig.10)
Table 2. Dimensions of different notch.

\begin{tabular}{|c|c|c|c|}
\hline Type & \multicolumn{3}{|c|}{ Base Length $(\mu \mathrm{m})$} \\
\hline Notch & \multicolumn{3}{|c|}{560} \\
\hline A & 250 & 450 & 610 \\
\hline B & 250 & 450 & 610 \\
\hline C & 250 & 450 & 610 \\
\hline D & 250 & 450 & 610 \\
\hline
\end{tabular}

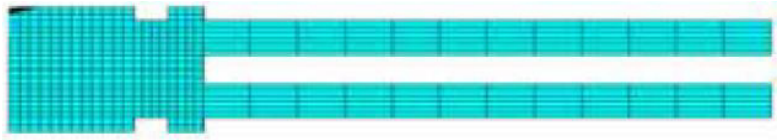

Base Length

Tine Length

Fig. 6. Modified tuning fork with notch.

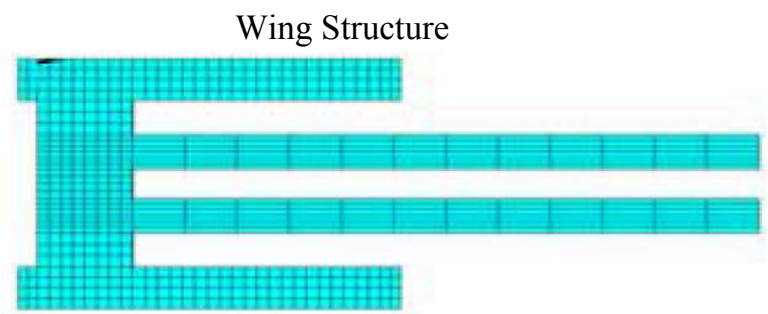

Base Length

Tine Length

Fig. 7. Tuning fork type A.

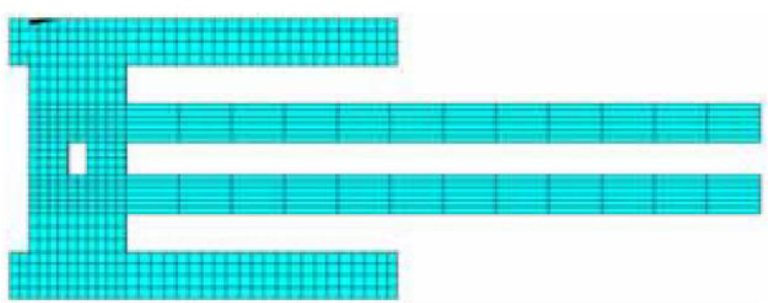

Base Length

Tine Length

Fig. 8. Tuning fork type B.

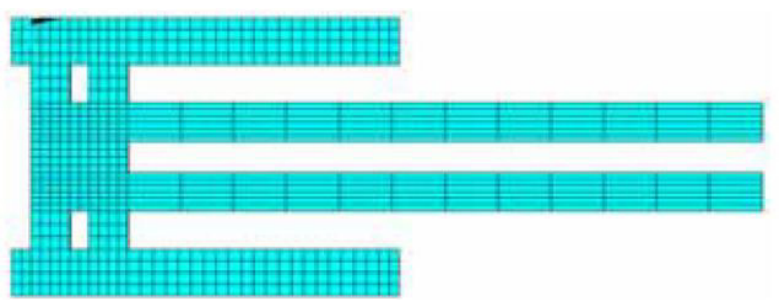

Base Length

Tine Length

Fig. 9. Tuning fork type C.

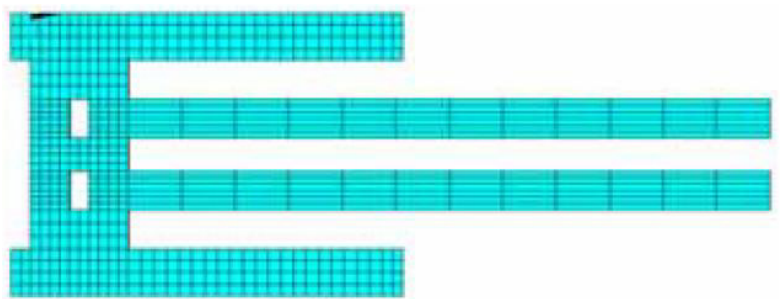

Base Length

Tine Length

Fig. 10. Tuning fork type D. 


\subsection{Results}

The electric response $(|\mathrm{Q}|$, Charge, fC) of type $\mathrm{A} \sim \mathrm{D}$ was calculated by harmonic analysis as follows (Fig. 11). Fig. 11 shows it is evident that electric response of model is better when base length is equal to $250 \mathrm{um}$. In case of base $250 \mathrm{um}$, type $\mathrm{C}$ is clearly better than other types, and location of hole shall be an important factor when base length is short. When base length is equal to 450 um or 610 um (Long base length), location of hole is not the important factor, but type B, C and D still have better electric response than type A.

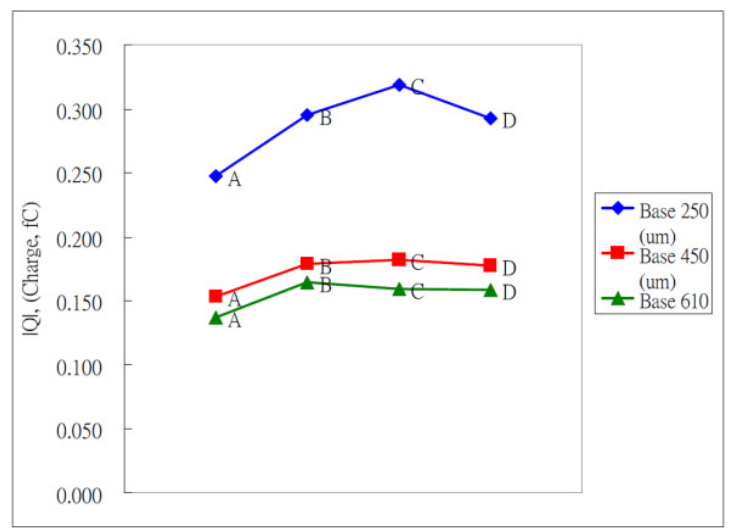

Fig. 11. Electric response.

Fig. 12 is that the result of type $\mathrm{A} \sim \mathrm{D}$ compares to modified tuning fork with notch. It is obvious that the type B, C and D with base length 250 um and "hole" are better than modified tuning fork with notch. The total length of type A, B, C and D with base length 250 um is $1890 \mathrm{um}$, it is shorter than modified tuning fork with notch (Total length: $2200 \mathrm{um}$ ).

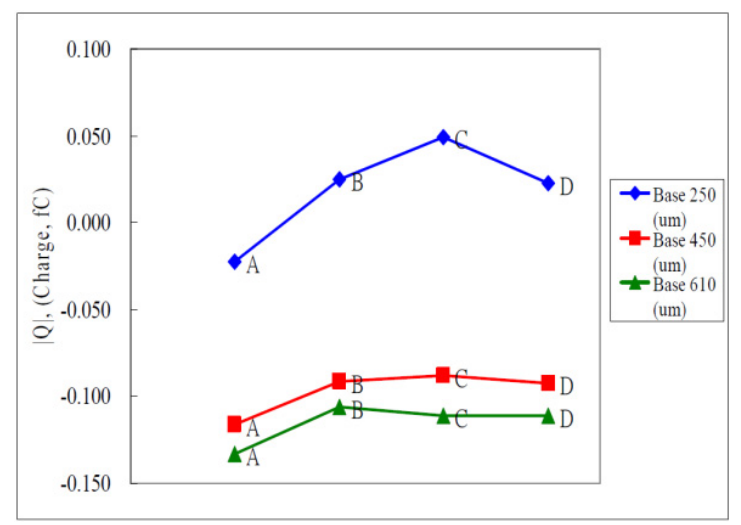

Fig. 12. Analytical results.

Fig. 13 shows that electric response is descent no matter what type is when base length is increasing, and it has an opposite trend to Fig. 4. However, type with hole really has better electric response than type without hole.

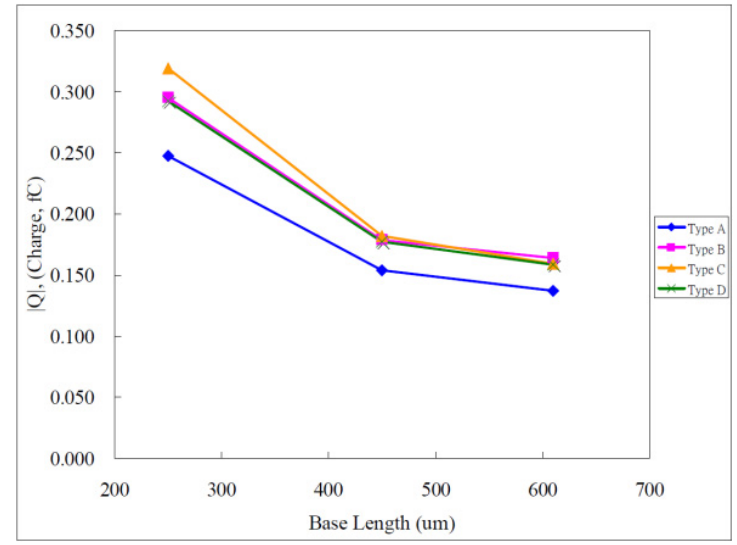

Fig. 13. Electric response.

\section{Conclusion}

In order to meet the restriction of tight space and well electric characteristic, several types of supporting structure of quartz tuning fork were discussed. The simulation result shows new supporting structure has a better electric response than modified tuning fork with notch, and hole located in base can effectively increase electric response. The author thinks hole may play a role of reflection mechanism to avoid vibration energy leakage to mounting areas, like beveling of AT-cut crystal. However, large hole may decrease stiffness of base. The balance between electric response and base stiffness needs to be confirmed.

Compare to modified tuning fork with notch, electric response is descent as base length of new supporting structure is increasing. This shall be a good info to miniaturization.

Next is to build models with electrode so to approach real device. The more complicated phenomenon can be involved in model to verify this view point. Experiment will be executed also.

\section{References}

1. J.A. Kusters, C.A. Adams, and H.E. Karrer and R.W. Ward, "Analytical and experimental investigations of $32 \mathrm{kHz}$ quartz tuning forks", Proc. $30^{\text {th }}$ Ann. Frequency Control Symp., 175-183(1976)

2. Hideaki Itoh and Takashi Matsumoto, "An analysis of frequency of a quartz crystal tuning fork by sezawa's approximation - The effect of clamped position of its base", Joint Meeting EFTF - IEEE IFCS,172-179(1999)

3. Shih S. Chuang, "Quartz tuning fork crystal using overtone flexure modes", Proc. $35^{\text {th }}$ Ann. Frequency Control Symp., 6-15(1981).

4. Yoshiro Tomikawa, Sumio Sugawara, and Masashi Konno , "Finite element analysis of displacement at base portion of a quartz crystal tuning fork", IEEE Transactions on Sonics and Ultrasonics, 26(3), 2329(1979) 
5. Yoshiro Tomikawa, Shigeru Oyama, and Masashi Konno, "A quartz crystal tuning fork with modified basewidth for a high quality factor: finite element analysis and experiments", IEEE Transactions on Sonics and Ultrasonics, 29(6),124-129(1982)

6. Fumitaka Kitamura, Junichiro Sakata and Hideo Tanaya, "US Patent 6587009”, 2003

7. Karl F. Graff, "Wave Motion in Elastic Solids", Dover Publications, Inc., New York

8. Roger Oria, Jorge Otero, Laura González, Luis Botaya, Manuel Carmona and Manel Puig-Vidal," Finite element analysis of electrically excited quartz tuning fork devices," Sensors, 13, 7156-7169 (2013)

9. M.R. Abdel-Rahman, B.A. Albassam, M.A. Alduraibi, " Driveless gyroscope response of a quatz piezoelctric vibratory tuning folk, " Acta Physica Polonica A, 127, 1352-1354 (2015)

10. Jorge Otero, Laura Gonzalez and Manel PuigVidal ,"Nanocharacterization of soft biological samples in shear mode with quartz tuning fork probes, "Sensors, 12, 4803-4819 (2012)

11. Anatoliy A. Kostereva and Frank K. Tittel, "Applications of quartz tuning forks in spectroscopic gas sensing," Review of Scientific Instruments, 76, 043105-1,043105-9 (2005)

12. Hideo Tanaya, "US Patent 20070024163”, 2007 